\title{
A Hybrid Optimization Method for Constrained Optimal Control Problem
}

\author{
Akbar Banitalebi, Mohd Ismail Abd Aziz, Rohanin Ahmad \\ Department of Mathematical Sciences, Faculty of Science, \\ Universiti Teknologi Malaysia, 81310 Skudai, Johor, Malaysia
}

\begin{abstract}
A new hybrid algorithm by integrating a nested partitions (NP) method with successive quadratic programming $(S Q P)$ is presented for global optimization of general optimal control problems involving lumped parameter system. The control parameterization technique first employed to reduce the control problem into a parameter selection problem. Then, in the global phase a vicinity of global optimizer is approximated by an appropriate NP method. Subsequently, the SQP algorithm in the local phase promotes the accuracy of final solution. The effectiveness of the hybrid NP-SQP algorithm is also illustrated by means of numerical simulations.
\end{abstract}

Keywords - Constraints, Nested partitions, Optimal control problem, Successive quadratic programming.

\section{INTRODUCTION}

In the early years of optimal control theory traditional methods based on calculus of variations could furnish some relatively simple problems with an analytical solution. As the analytical solution might not always be available, researchers have been interested in computational method for optimal control problems, yet when reliable and powerful computers became everywhere available, it was the end of an era. The software environments have become a platform for implementation of many practical methods to solve arising control problems. The iterative traditional methods both directly [3] or indirectly (using the Pontryagin's minimum principle) [8] rely on gradient information, and perform efficiently for convex problems, otherwise the performance of these methods highly depend on selected initial solution.

As an alternative to approximate a solution for complex nonconvex problems, many metaheuristic methods have been proposed [4]. In general, these methods iteratively sample amongst potential solutions, and the search is directed using the value of the objective function only, hence reducing the need for gradient information. Moreover, as metaheuristic methods globally search for optima, they are usually characterized as global optimizer due to their capability to efficiently arrive at the vicinity of a global optimum.

Nested partitions (NP) [10] is an exemplary method in this category, where concisely described in the third section. We use this method to globally search for optimal control for the class of the control problems described in the next section. Then, in the subsequent sections we propose the hybrid NP-SQP algorithm.

\section{THE CONTROL PROBLEMS}

In this study, we consider a general optimal control problem where the system of dynamics is described by a first-order ordinary differential equation (ODE),

$$
s(t)=f(x(t), u(t), t)
$$

with, $x\left(t_{0}\right)=x_{0}$ while the problem involves inequality constraints,

$$
C(x(t), u(t), t) £ 0 \text { for } t_{0} £ t £ t_{f} .
$$

The objective of the problem is then to identify the best control function $u$ to minimize a cost functional of the Mayer type $j\left(\mathrm{x}\left(\mathrm{t}_{\mathrm{f}}\right)\right)$ while the ODE (1) govern the interactions of problem variables, the Inequality (2) constricts them, and the control functions are additionally box-constrained, furthermore we assume there are constants $k$ and $l$ so that,

$$
\begin{gathered}
\|f(x(t), u(t), t)\| £ k(1+\|x(t)\|) \\
\|f(x(t), u(t), t)-f(y(t), u(t), t)\| £ l(\|x(t)-y(t)\|)
\end{gathered}
$$

satisfy for all feasible variables which guarantees a unique solution $(x \mid u)(t)$ or simply $x(t)$ for the ODE (1) (refer to [1]). We also assume all functions appear in the problem are continuously differentiable in the feasible region described by constraints; however this assumption is not required for global stage of optimization where NP method is used. 


\section{NP METHOD}

This method, as first proposed by Shi and Ólafsson [10], is an optimization algorithm for complex combinatorial problems where the search space is a finite set of potential solution, and the objective is to find an optimum solution to minimize or maximize a performance index The idea underlying NP method is to partition the search space into subspaces, and then the search procedure is started by sampling and evaluating a number of trial solutions from each subspace according to a fix sampling strategy.

Afterwards, a function $I: \Sigma \rightarrow \Re$ known as promising index function estimate the promising subspace. Then, the promising subspace is again partitioned into $M$ subspaces while the remainder subspaces are aggregated into one complementary subspace $s_{M+1}$. In the next iteration if $s_{M+1}$ is found to be promising then the algorithm backtracks to the state it has in the previous iteration. Therefore the algorithm, as explained above,

\section{NP Algorithm}

Step 0 Input the search space $\mathrm{Q}$, performance index $f$, promising index function $I$, the number of partitions $M$, initialize the whole search space to be promising $s^{*}(0)$, set the relevant stopping criterion, and set the iteration counter $k=1$.

Step 1 Partition the promising subspace $s^{*}(k-1)$ into $s_{i}^{*}(k)$ for $i=1, \mathrm{~K}, M$ subspace and set

$$
s_{M+1}(k)=\Theta-s^{*}(k-1)
$$

Step 2 Sample each $s_{i}(k), i=1, \mathrm{~K}, M$ according to a fix sampling strategy, and then evaluate sampled points.

Step 3 Estimate the promising subspace $s *(k)$ using the promising index function $I$.

Step 4 If $s *(k)=s_{M+1}(k-1)$ then backtrack by setting $s *(k+1)=s *(k-1)$, and $k=k-1$. If the stopping criterion is achieved, then stop the algorithm and output the best point sampled in the current promising subspace, otherwise set $k=k+1$ and go to Step 1 .

\section{NP-SQP ALGORITHM}

The hybrid NP-SQP algorithm uses the efficiency of NP method to search globally and SQP method for local search. The NP method can be applied when we use an equivalent constraint,

$$
\mathrm{O}_{t_{0}}^{t_{f}} \max (C(x(t), u(t), t), 0) d t=0,
$$

along with penalty technique. However, the NP algorithm is a combinatorial optimization method while the above problem is in continuous form. Therefore, this problem has first to be discretized. At this aim, the control parameterization technique is employed to approximate the control function with step functions of the form,

$$
u(t)=\stackrel{̊}{i=1}_{i}^{n} u_{i} c\left[t_{i}, t_{i+1}\right],
$$

where $c$ is indicator function, $t_{0} £ t_{1} £ \mathrm{~K} £ t_{n} £ t_{f}$ and the parameters $u_{i}$ are selected from a finite subset $D_{f}$ of a hypercube $D$ İ $\hat{\mathrm{A}}^{n}$. The subset $D_{f}$ can be obtained by uniformly discretizing each interval in hypercube $D$.

The NP method can now be applied to estimate a vicinity of global optimum for the resultant problem. The search space is first partitioned into a few numbers of subspaces. In the first iteration the values related only to the first parameter $u_{1}$ are partitioned and as the depth of the search is increased other parts of search space are also partitioned subsequently. Figure 1 shows partitioning in the situation where $n=4, u$ is one dimensional, $D=[-1,1]$ and $D_{f}$ is chosen to be $\{-1,0,1\}$. At the first iteration when the depth of the search is 1 , the search space is partitioned into three subspaces. Following that, in the next iterations the promising sub interval is partitioned with the same strategy.

$$
\begin{array}{ccc}
\text { depth }=1, & \{-1, X, X\},\{0, X, X\}, \text { and }\{1, X, X\} \\
\text { depth }=2, & \quad\{1,-1, X\},\{1,0, X\}, \text { and }\{1,1, X\} \\
\text { depth }=3, & \{1,-1,-1\},\{1,0,0\}, \text { and }\{1,1,1\}
\end{array}
$$

Fig. 1. An example of partitioning 
After running several iteration of NP algorithm, the global stage of NP-SQP algorithm is terminated and the best solution found is used as an initial solution for the local stage where SQP algorithm is employed to enhance the quality of solution.

As it is well known, the SQP algorithm is one of the most efficient algorithms for solving nonlinear and constrained programing problems and deeply studied by many authors see $[2,7,9,14]$ and the references therein. This algorithm successively approximates the constrained nonlinear problem with quadratic problem and linear constraint to direct the search and therefore a proper initial solution as well as gradient information is required. The initial solution is supplied by running few iterations of NP algorithm, and the gradient with respect to parameters $u_{i}$ can be found in [11]. In this book, gradient information was presented for unified form of optimal control problem. However the Constraint (3) in the problem is nonsmooth, therefore the following function is used as a smoothing technique,

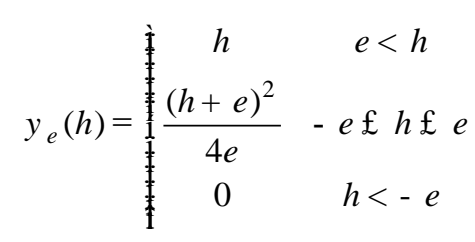

where, the parameter $e$ can be any small enough positive real number. This function was used in [12] to approximate nonsmooth problems. The previously described problem can then be approximated as well with the same approach as follow,

$$
\begin{gathered}
\min \mathrm{J}=j\left(\mathrm{x}\left(\mathrm{t}_{\mathrm{f}}\right)\right) \\
\text { subject to } \grave{\mathrm{O}}_{t_{0}}^{t_{f}} y_{e}(C(x(t), u(t), t)) d t=0
\end{gathered}
$$

therefore, SQP algorithm with quasi-Newton Jacobian updates for equality constrained problems can be readily applied on Problem (4) [7]. As a result, the solution found by NP method, after a few iterations, is improved upon to the required accuracy, and this terminates the local stage of the hybrid algorithm.

\section{NUMERICAL RESULTS}

In this section, the effectiveness of the NP-SQP algorithm described above is illustrated using the following problem,

$$
\begin{aligned}
& \min x_{3}\left(t_{f}\right) \\
& \quad \& \&=x_{2} \\
& x \&(t)=-x_{2}(t)+u(t) \\
& x \&(t)=x_{1}^{2}(t)+x_{2}^{2}(t)+0.005 u^{2}(t) \\
& x_{1}(t)-8(t-0.5)^{2}+0.5 £ 0
\end{aligned}
$$

while, $0 £ t £ 1, \quad-5 £ u(t) £ 5$ and $x(0)=[0,-1,0]^{T}$.

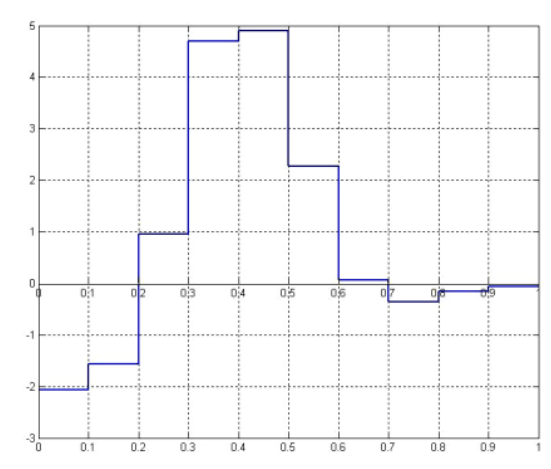

Fig. 2. The optimal control

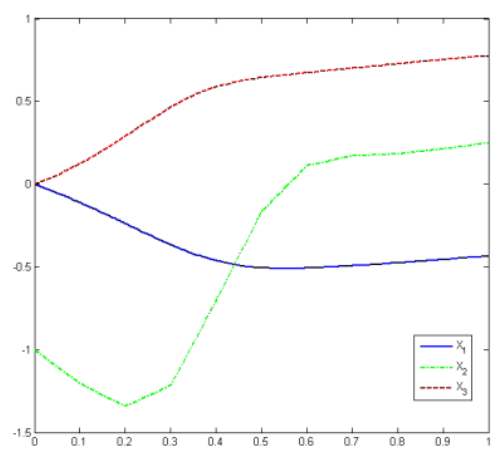

Fig. 3. The optimal states 
This problem frequently used as a benchmark problem and solved by Neuman and Sen [6], Goh and Teo [5], and Vlassenbroeck [13]. This problem was first transformed into the form of Problem (4), and then after ten iterations of NP algorithm the best solution $J_{N P}^{*}=0.7775$ was used as initial solution for SQP algorithm. The previous solution then was slightly improved to $J^{*}=0.7641$. The graph of optimal control as well as related graphs of state is available in Figures 2 to 4 . These results agree with the one published in $[6,13]$.

\section{CONCLUSION}

We presented a hybrid two-stage algorithm where in global stage NP method along with control parameterization technique and penalty method were used to estimate a global optimum for the problem under consideration. Afterwards, this solution was used as an initial solution in the next stage of optimization, where an SQP algorithm with quasi newton for equality constraint problems is employed to enhance the quality of solution. The method was evaluated using a benchmark problem, and it was revealed that the algorithm is effective.

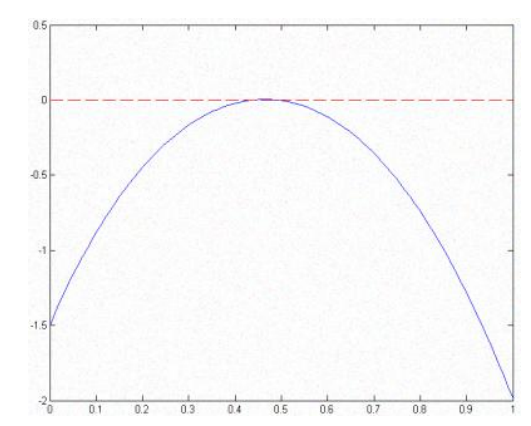

Fig. 4. The constraint violations

\section{Acknowledgements}

This study supported by the Malaysian Ministry of Higher Education and Research Management Centre of Universiti Teknologi Malaysia through research grant vot. no. 4B045.

\section{REFERENCES}

[1] N. U., Ahmed, Dynamic systems and control with application. World Scientific, Singapore, (2006).

[2] A., Antoniou, W. S., Lu, Practical Optimization: Algorithms and Engineering Applications. Springer, New York, (2007).

[3] E., Polak, An historical survey of computational methods in optimal control, Siam Rev. 15 (1973), no. 2, 553-584.

[4] F., Glover, G. A., Kochenberger, Handbook of metaheuristics, Kluwer academic publishers. Dordrecht, Netherlands, (2003).

[5] C. J., Goh, K. L., Teo, Control parametrization: A unified approach to optimal control problems with general constraints. Automatica 24 (1988), no. 1, 3-18.

[6] C. P., Neuman, A., Sen, A suboptimal control algorithm for constrained problems using cubic splines. Automatica 9 (1973), 601613 .

[7] J., Nocedal, S. J., Wright, Numerical Optimization. 2nd Ed, Springer, New York, USA, (2006).

[8] L. S., Pontryagin, V. G., Boltyanskii, R. V., Gamkrelidze, and E. F., Mischenko, The Mathematical Theory of Optimal Processes (Translation by L. W., Neustadt), Macmillan, New York, USA, (1962).

[9] M. J. D., Powell, Y., Yuan, A recursive quadratic programming algorithm that uses differentiable exact penalty functions, Math. Program. 35 (1986) 265-278.

[10] L., Shi, S., Ólafsson, Nested partitions method for global optimization. Oper. Res. 48 (2000), no. 3, $390-407$.

[11] K. L., Teo, C. J. Goh, and K. H., Wong, A unified computational approach to optimal control problems. Longman Scientific and Technical, (1991).

[12] K. L., Teo, V., Rehbock, and L. S., Jennings, A new computational algorithm for functional inequality constrained optimization problems. Automatica 29 (1993), no. 3, 789-792.

[13] J., Vlassenbroeck, A Chebyshev polynomial method for optimal control with state constraints. Automatica 24 (1988), no. 4, 499506.

[14] B. Yang, K. Zhang, Z. You, A successive quadratic programming method that uses new corrections for search directions, J. Comput. Appl. Math. 71 (1996) 15-31. 